\title{
An Evolutionary Algorithm for the Synthesis of Oblique Incidence Optical Coatings
}

\author{
Jinn-Moon Yang \\ Department of CSIE \\ National Taiwan University \\ Taipei 106, Taiwan \\ moon@csie.ntu.edu.tw
}

\author{
Cheng-Yan Kao \\ Department of CSIE \\ National Taiwan University \\ Taipei 106, Taiwan \\ cykao@csie.ntu.edu.tw
}

\begin{abstract}
This paper presents an evolutionary approach for the aynthesis of oblique incidence optical thin-film designs. Based on tamily competition and adaptive rules, the proposed approach consists of global and local strategies by integrating decreaning mutations and relf-adaptive mutations. The method is applied to a called heat reflector and a cold mirror in the region from $0.4 \mu \mathrm{m}$ to $1.2 \mu \mathrm{m}$ with the incidence angle $45^{\circ}$. The proposed approach generates binary-type solutions which are very close to the target apecification. Experimental results show that the proposed approach is very flexible and performs very robustly.
\end{abstract}

\section{INTRODUCTION}

There is a need for many different thin-film design approaches which are often required for the solutions of different types of problems. These approaches can be roughly classified into analytical, graphical, and numerical methods [6], [10]. The numerical method is now the most widely used design technique. It is particularly powerful because it can be applied to the design of coatings with much more complicated properties than is the possible with the other methods. In most numerical methods the design of optical coatings is formulated as an optimization problem based on the use of merit functions. Nevertheless, in many cases the numerical solutions of the resulting optimization problems are extremely difficult because of the large number of local minimum in the merit function.

The two basic approaches to the design of numerical optical coatings are refinement methods [1] and synthesis methods [9], [14]. Refinement methods normally require a starting design that is not quite satisfactory. And then a desired solution is achieved by gradually modifying from the staring design. The solution quality of refinement methods is sensitive to the start point. Unfortunately, good starting designs are not readily available for many design problems. At the same time, to choose a good starting design is time-consuming and difficult tasks in a complexity coating system. Contrary to refinement methods, synthesis methods [9] generate their own starting designs automatically. They usually combine with numerical refinement methods due to their solution quality is often not good enough. Some of synthesis and refinement methods require the determination of first and second derivation of the merit function. Therefore, to develop a good synthesis method is an important research topic.

Recently evolutionary algorithms [11], [8] have success- fully applied to many problems encountered in optical filters and coatings which are inherently computational complexity. These articles demonstrated that evolutionary algorithms are competitive with well-known synthesis methods. An evolutionary algorithm is a generally adaptable concept for problem solving, especially well suited for solving difficult optimization problems. It is based on the ideas borrowed from genetics and natural selection. Evolutionary algorithms have been used to solve problems involving large search spaces, where traditional optimization methods are less efficient.

There are about three main independently developed but strongly related implementations of evolutionary algorithms: genetic algorithms, evolution strategies [2], and evolutionary programming [7]. For genetic algorithms, both practice and theory, entail disadvantages of applying binary-represented implementation to the design of optical coatings. The coding function of binary-represented genetic algorithms may introduce an additional multimodality, making the combined objective function more complex than the original function. To achieve better performance, real-coded genetic algorithms [5] have been introduced. In contrast, evolution strategies [8], [12] and evolutionary programming mainly use real-valued representation and focus on self-adaptive Gaussian mutations to the design of optical coatings. This type of mutation has succeeded in continuous optimization and has been widely regarded as a good operator for local searches. Unfortunately, experiments show that self-adaptive Gaussian mutation leaves individuals trapped near local optima for rugged functions.

In this paper we use a method called family competition evolutionary algorithm (FCEA) to synthesize optical thinfilm systems with various number of layers. FCEA has been successfully applied to solve global optimization [18] and flexible ligand docking [17]. The proposed approach combines decreasing-based Gaussian mutation, self-adaptive Gaussian mutation, and self-adaptive Cauchy mutation. It incorporates family competition [16] and adaptive rules to construct the relationship between mutations whose performance heavily depends on the same factor, called step size. The self-adaptive mutations adapts the step sizes with a stochastic mechanism and decreasing-based mutations decrease the step sizes with a fixed rate $\gamma$ where $\gamma<1$. In order to balance exploration and exploitation, each of these operators is designed to cooperate with one another. The 


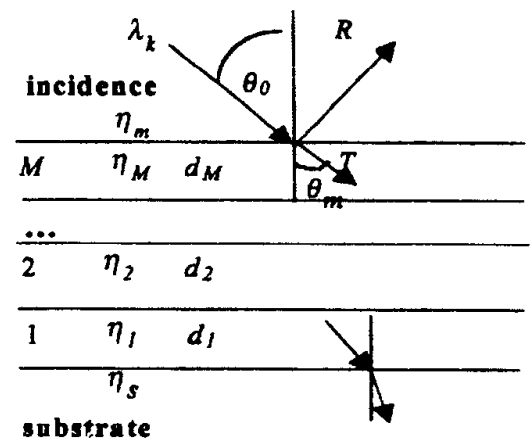

Fig. 1. The construction parameters of an optical coating system. $\theta_{0}$ is the incident angle. $\eta_{j}$ and $d_{j}$ are the refractive index and thicknese of the $j$ th layer where $1 \leq j \leq M$. The $\eta_{m}$ and $\eta_{m}$ denote the incidence and substrate refractive indices, respectively.

family competition is inspired from $(1+\lambda)$-ES [2] and is similar to a local search procedure.

The rest of this paper is organized as follows. Section II describes the problem of optical thin-film coatings. Section III introduces the evolutionary nature of FCEA. In Section IV the synthesis of two edge filters are presented to illustrate the performance of our proposed approach. Concluding comments are drawn in Section V.

\section{Problem Definition}

The problem in numerical design of optical multilayer coatings is to find the construction parameters of a system which satisfies the desired optical specification. The construction parameters includes the number of layers $(M)$; the thickness $(d)$ and refractive indices $(\eta)$ of the medium, substrate, and layers. Fig. 1 shows a M-layer coating system.

To design a multilayer coating system, it is necessary to define the desired optical specification. Most often this is defined by specifying the target transmittance $\hat{T}$ or target reflectance $R$ at a number of wavelengths in the interesting spectral region. $\hat{T}$ equals $1-\hat{R}$ if the materials of a multilayer coating system are all nonabsorbing. Designing a thin-film system is to find the number of layers $M$, the refractive indices $\eta_{j}$, and the thickness $d_{j}$ of the $j$ th layer, in order to match closely the specified performance where $1 \leq j \leq M$. A coating system is called normal-incidence coating if the incident angle $\theta_{0}$ shown in Fig. 1 is zero, otherwise it is called oblique-incidence coating. In this paper, we consider the oblique-incidence coating and the materials are nonabsorbing.

Let the spectral reflectance of the $M$-layer system shown in Fig. 1 be denoted as $R(\eta, d, \lambda)$ where $\lambda$ is the interesting wavelength region. A widely working merit function is given

$$
f\left(\eta, d, \lambda_{k}\right)=\frac{1}{W} \Sigma_{k=1}^{W}\left\{\frac{\left[R\left(\eta, d, \lambda_{k}\right)-\hat{R}\left(\lambda_{k}\right)\right]^{2}}{\delta R_{k}}\right\}^{1 / 2},
$$

where $R\left(\eta, d, \lambda_{k}\right)$ and $\hat{R}\left(\lambda_{k}\right)$ are respectively the desired and the target reflectance at wavelength $\lambda_{k} ; \lambda_{k}$ are wavelength points from a given wavelength grid with the total

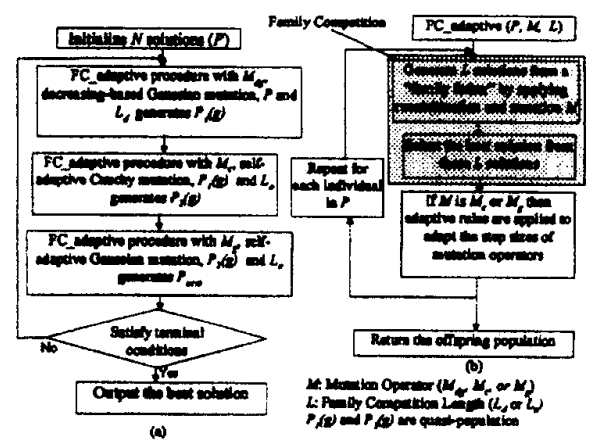

Fig. 2. Overview of our algorithm: (a) FCEA (b) FC_adaptive procedure

number of $M$ points, and $\delta R_{k}$ is the tolerance at wavelength $\lambda_{k}$; In general the value of $\delta R_{k}$ is set to 0.01 . The most general method of calculating $R\left(\eta, d, \lambda_{k}\right)$ is based on a matrix formulation which is useful especially when the number of optimizing parameters is large [6].

According to the maximum principle [14], it is no advantage to use more than two materials that have the lowest $\eta_{l}$ and highest $\eta_{h}$ refractive indices at normal light incidence. That is, the best results will be achieved with the pair of materials having the lowest and highest refractive indices. Second, the number of layers may be limited because the cost of coatings increases with the number of layers. $\mathrm{Fi}$ nally, the thickness cannot be negative as well as very thin layers are difficult controlled for some deposition processes. In this paper, a layer will be eliminated if its thickness is lower $0.001 \mu \mathrm{m}$. We also use only one pair of materials with $\eta_{l}$ and $\eta_{h}$ for the design of optical coatings.

\section{Family Competition Evolutionafy Algorithm}

In this section, we present the detail of the family competition evolutionary algorithm (FCEA) for the optical thin-film deigns. The basic structure of the FCEA is as follows (Fig. 2): $N$ solutions are randomly generated as the initial population. Then FCEA enters the main evolutionary loop, in which each generation consists of three nearly identical procedures. Each procedure is realized by doing recombinations, mutations, family competition, and selection. These three procedures differ mainly in the mutations used: decreasing Gaussian mutation $\left(M_{d g}\right)$, selfadaptive Cauchy mutation $\left(M_{c}\right)$, and self-adaptive Gaussian mutation $\left(M_{g}\right)$. Hence we refer such a procedure as "FC_adaptive" which will be described later in detail. The output is a new quasi-population with $N$ solutions which will be the input of the next FC_adaptive procedure.

The FC_adaptive procedure employs three parameters, namely, the parent population ( $P$, with $N$ solutions), mutation operator $(M)$, and family competition length $(L)$, to generate a new quasi-population (Fig. 2(b)). The "FC_adaptive" procedure proceeds as follows to generate a quasi-population: Each individual in the population sequentially becomes the "family father." With a probability $p_{c}$, this family father and another solution randomly chosen from the rest of the parent population are used as parents 
to do a recombination operation. Then the new offspring or the family father (if the recombination is not conducted) is operated on by a mutation. For each family father, such a procedure is repeated $L$ times. Finally $L$ children are produced but only the one with the best objective value survives. Since we create $L$ children from one "family father" and perform a selection, this is a family competition strategy. After the family competition, there are $N$ parents and $N$ children left. In each pair of father and child the individual with a better objective value survives. This is called "family selection."

Regarding chromosome representation, we present each solution of a population as $(M, I, x, \sigma, v, \psi)$ where $M$ is the number of layers of a coating system. We use a indicator $I$ to represent the structure of the refractive indices of a coating system because we use only one pair of materials with $\eta_{l}$ and $\eta_{h}$. The refractive index of first layer is equivalent to $\eta_{h}$ when $I$ is 0 and it is equivalent to $\eta_{h}$ when $I$ is 1 . The vector $x$ is the thickness vector of a coating system to be optimized; $\sigma, v$, and $\psi$ are the step-size vectors of decreasing-based mutation, self-adaptive Gaussian mutation, and self-adaptive Cauchy mutation, respectively. In other words, each solution $x$ is associated with some parameters for step-size control. The number of element of each vector, $x, \sigma, v$, and $\psi$, is $M$. The initial value $M$ is randomly chosen from $\left[M_{l}, M_{h}\right]$ where $M_{l}$ and $M_{h}$ are the numbers of the low bound and upper bound layers, respectively. $I$ is randomly set to 1 or 0 . The initial value of each entry of $x$ is randomly chosen over a feasible region depended on the properties of the specification of optical coatings. The initial values of each entries of $\sigma, v$, and $\psi$ are set to $0.04,0.01$, and 0.01 .

In the rest of this section we explain each important component of the FC adaptive procedure: recombination operators, mutation operations, and rules for adapting step sizes $(\sigma, v$, and $\psi)$. For easy description of the operators, we use $a=\left(M^{a}, I^{a}, x^{a}, \sigma^{a}, v^{a}, \psi^{a}\right)$ to represent the "family father" and $b=\left(M^{b}, I^{b}, x^{b}, \sigma^{b}, v^{b}, \psi^{b}\right)$ as another parent (only for the recombination operator). The offspring, $c=\left(M^{c}, I^{c}, x^{c}, \sigma^{c}, v^{c}, \psi^{c}\right)$, is generated by a genetic operation. We also use the symbol $x_{j}^{d}$ to denote the $j$ th component of an individual $d, \forall j \in\{1, \ldots, M\}$.

\section{A. Recombination Operators}

The advantages or disadvantages of recombination for a particular objective function can hardly be accessed in advance [3]. Therefore, we implement two simple recombination operators to generate offspring: modified discrete recombination and intermediate recombination [2]. With probabilities 0.8 and 0.2 , at each FC_adaptive procedure only one of the two operators is chosen. Probabilities are set according to our experimental experience. Here we would like to mention again that recombination operators are activated with only a probability $p_{c}$.

Modifled Discrete Recombination: The original discrete recombination [2] generates a child that inherits genes from two parents with equal probability. Here the two parents of the recombination operator are the "family father" and another solution randomly selected. Our experience indicates that FCEA can be more robust if the child inherits genes from the "family father" with a higher probability. Therefore, we modified the operator to be as follows:

$$
x_{j}^{c}=\left\{\begin{array}{l}
x_{\dot{j}}^{a} \text { with probability } 0.8 \\
x_{j}^{b} \text { with probability } 0.2 .
\end{array}\right.
$$

For a "family father", applying this operator in the family competition is viewed as a local search procedure be cause this operator is designed to preserve the relationship between a child and its "family father".

Intermediate Recombination: We define intermediate recombination as:

$$
\begin{aligned}
x_{j}^{c} & =x_{j}^{a}+0.5\left(x_{j}^{b}-x_{j}^{a}\right), \text { and } \\
w_{j}^{c} & =w_{j}^{a}+0.5\left(w_{j}^{b}-w_{j}^{a}\right),
\end{aligned}
$$

where $w$ is $v, \sigma$, or $\psi$ based on the mutation operator applied in the family competition. For example, if selfadaptive Gaussian mutation is used in this FC adaptive procedure, $x$ in (3) and (4) is $v$. We follow the work of the evolution strategies community [4] to employ only intermediate recombination on step-size vectors, that is, $\sigma, v$, and $\psi$.

\section{B. Mutation Operators}

Mutations are main operators of the FCEA. After the recombination, a mutation operator is applied to the "family father" or the new offspring generated by a recombination. In FCEA, the mutation is performed independently on each vector element of the selected individual by adding a random value with expectation zero:

$$
x_{i}^{\prime}=x_{i}+w D(\cdot),
$$

where $x_{i}$ is the thickness of the $i$ th of $x, x_{i}^{\prime}$ is the $i$ th variable of $x^{\prime}$ mutated from $x, D(\cdot)$ is a random variable, and $w$ is the step size. In this paper, $D(\cdot)$ is evaluated as $N(0,1)$ or $C(1)$ if the mutations are, respectively, Gaussian mutation or Cauchy mutation.

Self-Adaptive Gaussian Mutation: We adapted Schwefel's [13] proposal to use self-adaptive Gaussian mutation. The mutation is accomplished by first mutating the step size $v_{j}$ and then the thickness $x_{j}$ :

$$
\begin{aligned}
v_{j}^{c} & =v_{j}^{a} \exp \left[\tau^{\prime} N(0,1)+\tau N_{j}(0,1)\right], \\
x_{j}^{c} & =x_{j}^{a}+v_{j}^{c} N_{j}(0,1),
\end{aligned}
$$

where $N(0,1)$ is the standard normal distribution. $N_{j}(0,1)$ is a new value with distribution $N(0,1)$ that must be regenerated for each index $j$. For FCEA, we follow [4] in setting $\tau$ and $\tau^{\prime}$ as $(\sqrt{2 n})^{-1}$ and $(\sqrt{2 \sqrt{n}})^{-1}$, respectively.

Self-Adaptive Cauchy Mutation: We follow previous works [19] to define self-adaptive Cauchy mutation as follows:

$$
\begin{aligned}
\psi_{j}^{c} & =\psi_{j}^{a} \exp \left[\tau^{\prime} N(0,1)+\tau N_{j}(0,1)\right] \\
x_{j}^{c} & =x_{j}^{a}+\psi_{j}^{c} C_{j}(t) .
\end{aligned}
$$




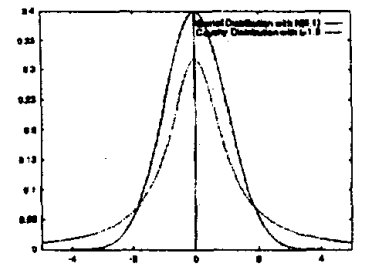

Fig. 3. Density functions of Gaussian and Cauchy distributions.

In our experiments, $t$ is 1 . Note that self-adaptive Cauchy mutation is similar to self-adaptive Gaussian mutation except that (7) is replaced by (9). That is, they implement the same step-size control but use different means of updating $x$.

Decreasing-Based Gaussian Mutations: Our decreasing-based Gaussian mutation uses the step-size vector $\sigma$ with a fixed decreasing rate $\gamma=0.97$ as follows:

$$
\begin{aligned}
& \sigma^{c}=\gamma \sigma^{a} \\
& x_{j}^{c}=x_{j}^{a}+\sigma^{c} N_{j}(0,1)
\end{aligned}
$$

Previous results [18] demonstrated that self-adaptive mutations converge faster than decreasing-based mutations but, for rugged functions, self-adaptive mutations more easily trapped into local optima than decreasing-based mutations.

Fig. 3 compares density functions of Gaussian distribution $(N(0,1))$ and Cauchy distributions $(C(1))$. Clearly Cauchy mutation is able to make a larger perturbation than Gaussian mutation. This implies that Cauchy mutation has a higher probability of escaping from local optima than Gaussian mutation does. The detailed reasons why we want to use these three types of mutations can be referred [18].

\section{Adaptive Rules}

The performance of Gaussian and Cauchy mutations is largely influenced by the step sizes. FCEA adjusts the step sizes while mutations are applied (e.g. (6), (8), and (10)). However, such updates insufficiently consider the performance of the whole family. Therefore, after family competition, some additional rules are implemented:

1. A-decrease-rule: Immediately after self-adaptive mutations, if objective values of all offspring are greater than or equal to that of the "family parent," we decrease the step-size vectors $v$ (Gaussian) or $\psi$ (Cauchy) of the parent:

$$
w_{j}^{a}=0.95 w_{j}^{a},
$$

where $w^{a}$ is the step size vector of the parent. In other words, when there is no improvement after self-adaptive mutations, we may propose that a more conservative, that is, smaller, step size tends to make better improvement in the next iteration.

2. D-increase-rule: It is difficult, however, to decide the rate $\gamma$ of decreasing mutations. Unlike self-adaptive mutations which adjust step sizes automatically, its step size

\begin{tabular}{|c|c|}
\hline parameter name & the value and notation of parameter \\
\hline $\begin{array}{l}\text { recombination } \\
\text { probability }\left(p_{c}\right)\end{array}$ & $\begin{array}{l}P_{c D}=0.8\left(\text { for } M_{d g}\right), \\
p_{c A}=0.2 \text { (for } M_{c} \text { or } M_{f} \text { ). }\end{array}$ \\
\hline $\begin{array}{l}\text { family competition } \\
\text { length }\end{array}$ & 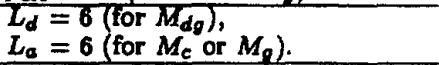 \\
\hline step Biges & $v_{i}=\phi_{i}=0.01, \sigma_{i}=4 v_{i}$ \\
\hline population size $(N)$ & 50 \\
\hline other notation & $\begin{array}{l}\text { M: number of lavers, } \\
\text { MF: value of merit function, } \\
\eta d \text { : total thickness of a solution. }\end{array}$ \\
\hline
\end{tabular}

TABLE I

PARAMETERS OF FCEA AND NOTATION USED IN THIS PAPER

goes to zero as the number of generations increases. Therefore, it is essential to employ a rule which can enlarge the step size in some situations. The step size of the decreasing mutation should not be too small, when compared to step sizes of self-adaptive mutations. Here, we propose to increase $\sigma$ if one of the two self-adaptive mutations generates better offspring. To be more precise, after a self-adaptive mutation, if the best child with step size $v$ is better than its "family father," the step size of the decreasing mutation is updated as follows:

$$
\sigma^{c}=\max \left(\sigma^{c}, \beta v_{\text {mean }}^{c}\right),
$$

where $v_{\text {mean }}^{c}$ is the mean value of the vector $v$; and $\beta$ is 0.2 in our experiments. Note that this rule is applied in stages of self-adaptive mutations but not of decreasing mutations.

\section{EXPERIMENTAL RESULTS}

In this section we present the numerical results for the synthesis of two edge filters to illustrate the proposed method. Table I indicates the setting of FCEA parameters, such as initial step sizes, family competition lengths and recombination probabilities. They are used for synthesis problems defined in this work. $L_{d}, \sigma$, and $p_{c D}$ are the parameters for decreasing-based mutation; $L_{a}, v, \psi$, and $p_{c A}$ are for self-adaptive mutations. The population size is 50. These parameters are decided after experiments on these two optical coatings with various values. For each problem FCEA is tested 30 times.

\section{A. Long-wave-pass filter}

The second design problem is the synthesis of a longwave-pass filter for $s-$ and $p$-polarized light at $45^{\circ}$. Fig. 4(a) indicates the target specification. The filter is a cold mirror which reflects as much as possible of the visible light incident upon them and transmits the remaining radiation. Both the $s$ and $p$ cases the targets were 1.0 reflectance in the region from 0.4 to $0.8 \mu \mathrm{m}$ and the reflectance was zero in the region from 0.85 to $1.2 \mu \mathrm{m}$. The incident medium is air $\left(\eta_{m}=1\right)$ and the substrate is glass with $\eta_{c}=1.52$. The high- and the low-index refractive indices were $\eta_{h}=2.35$ and $\eta_{l}=1.35$, respectively. The merit function was also defined at 39 points.

The initial number of layers is randomly chosen from 25 to 60 . The initial thickness of each layer was uniformly selected from the region from 0.01 to $0.1 \mu \mathrm{m}$. The maximum number of generations is 1500 . 


\section{TABLE II}

CONSTRUCTION PARAMETERS OF THE \&-POLARIzATION OF THE SHORT-WAVE-PASS FILTER AND OF THE LONG-WAVE-PASS FLLTER.

\begin{tabular}{|c|c|c|c|c|}
\hline Layer & \multicolumn{2}{|c|}{$\begin{array}{l}\text { short-wave-pess filter } \\
\text { s-polarization }\end{array}$} & \multicolumn{2}{|c|}{$\begin{array}{c}\text { Tong-wave-pass filter } \\
\text { - - polarization }\end{array}$} \\
\hline Subs(nt) & & & & \\
\hline 1 & 2.35 & 0.01631 & 1.45 & 0.44943 \\
\hline 2 & 1.45 & 0.01814 & 2.35 & 0.05743 \\
\hline 3 & 2.35 & 0.11283 & 1.45 & 0.06604 \\
\hline 4 & 1.45 & 0.20981 & 2.35 & 0.12593 \\
\hline 5 & 2.35 & 0.11822 & 1.45 & 0.08192 \\
\hline 6 & 1.45 & 0.23115 & 2.35 & 0.09485 \\
\hline 7 & 2.35 & 0.01001 & 1.45 & 0.10638 \\
\hline 8 & 1.45 & 0.02222 & 2.35 & 0.07607 \\
\hline 9 & 2.35 & 0.01002 & 1.45 & 0.13735 \\
\hline 10 & 1.45 & 0.22033 & 2.35 & 0.08491 \\
\hline 11 & 2.35 & 0.11273 & 1.45 & 0.11492 \\
\hline 12 & 1.45 & 0.20554 & 2.35 & 0.07759 \\
\hline 13 & 2.35 & 0.13547 & 1.45 & 0.08634 \\
\hline 14 & 1.45 & 0.02614 & 2.35 & 0.15958 \\
\hline 15 & 2.35 & 0.13397 & 1.45 & 0.08878 \\
\hline 16 & 1.45 & 0.20600 & 2.35 & 0.05881 \\
\hline 17 & 2.35 & 0.11738 & 1.45 & 0.11170 \\
\hline 18 & 1.45 & 0.23381 & 2.35 & 0.17752 \\
\hline 19 & 2.35 & 0.03280 & 1.45 & 0.06772 \\
\hline 20 & 1.45 & 0.04522 & 2.35 & 0.05008 \\
\hline 21 & 2.35 & 0.03005 & 1.45 & 0.23398 \\
\hline 22 & 1.45 & 0.24562 & 2.35 & 0.04599 \\
\hline 23 & 2.35 & 0.01418 & 1.45 & 0.09472 \\
\hline 24 & 1.45 & 0.01463 & 2.35 & 0.15562 \\
\hline 25 & 2.35 & 0.11425 & 1.45 & 0.08259 \\
\hline 26 & 1.45 & 0.21041 & 2.35 & 0.04518 \\
\hline 27 & 2.35 & 0.11237 & 1.45 & 0.17860 \\
\hline 28 & 1.45 & 0.20064 & 2.35 & 0.06825 \\
\hline 29 & 2.35 & 0.10941 & 1.45 & 0.05959 \\
\hline 30 & 1.45 & 0.19994 & 2.35 & 0.06566 \\
\hline & 2.35 & 0.10621 & 1.45 & 0.15079 \\
\hline 32 & 1.45 & 0.09507 & 2.35 & 0.03476 \\
\hline 33 & & & 1.45 & 0.09478 \\
\hline 34 & & & 2.35 & 0.13713 \\
\hline 35 & & & 1.45 & 0.15067 \\
\hline & & & 2.35 & 0.01190 \\
\hline medium $\left(\eta_{m}\right)$ & 1.0 & & 1.0 & \\
\hline$\sum n d$ & & 6.48 & & 7.0 \\
\hline $\operatorname{MF}(\%)$ & & 1.019 & & 0.279 \\
\hline
\end{tabular}

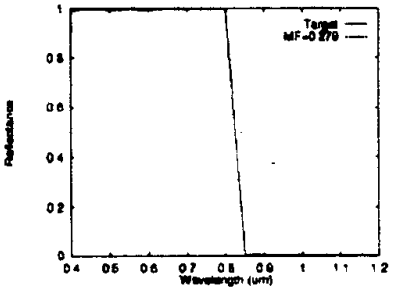

(a) Reflectance

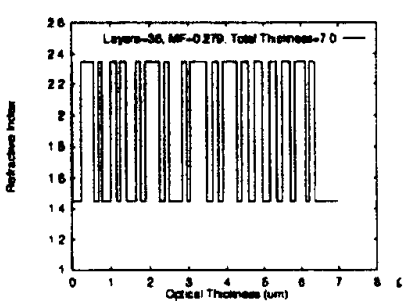

(b) Reflective index profile
Fig. 4. Reflectance and refractive-index profile of a 36-layer longwave-pass filter for s-polarized light at a $45^{\circ}$ angle of incidence on interesting region $0.4 \leq \lambda \leq 1.2 \mu \mathrm{m}$ on a $\eta_{0}=1.52$ substrate based on the refractive index pair 1.45 and 2.35 .

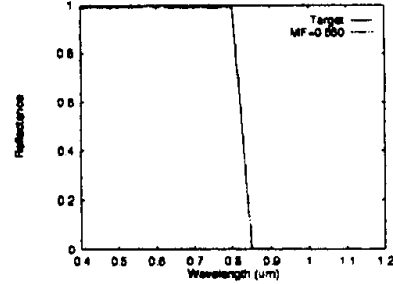

(a) Refectance

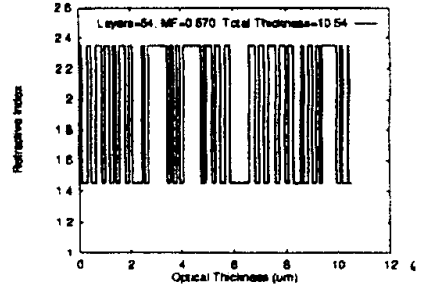

(b) Reflective index profile
Fig. 5. Reflectapce and refractive-index profile of a 54-layer longwave-pass filter for p-polarized light at a $45^{\circ}$ angle of incidence on interesting region $0.4 \leq \lambda \leq 1.2 \mu \mathrm{m}$ on a $\eta_{s}=1.52$ substrate based on the refractive index pair 1.45 and 2.35 .

Fig. 4 shows the reflectance and the refractive-index profile of s-polarized case of a final coating system whose number of layers is 36 and the value of merit function is $0.279 \%$. The total thickness of this solution is $7.0 \mu \mathrm{m}$. The final solution of the $p$-polarized case is shown in Fig. 5. Its total thickness is $10.54 \mu \mathrm{m}$. The number of layers and MF of this final solution is 54 and $0.570 \%$, respectively. Obviously, the total thickness of the final design in the $p$ polarized case and the number of layers are considerably more than in the 8 -polarized case. The construction parameters of a 8-polarized solution generated by FCEA are given in the final column of Table II. According to the experimental results, FCEA is able to obtain coating systems which are close to the target specification.

\section{B. Short-wave-pass filter}

The first problem is the synthesis of a short-wave-pass filter for $8-$ and $p$-polarized light at $45^{\circ}$ [15]. The target reflectance of the specification is the solid curve shown in Fig. 6(a). The filter is a heat reflector (or called hot mirror) which is a special long-wavelength cut-off filter in a cut-off at $0.8 \mu \mathrm{m}$. That is, the filter transmits the visible radiation from 0.4 to $0.8 \mu \mathrm{m}$ without disturbing the color balance. The width of the rejection region depends on the light source to be used. In this case both the $s$ and $p$ cases the targets were zero reflectance in the region from 0.4 to $0.8 \mu \mathrm{m}$ and the refectance was 1.0 in the region from 0.85 to $1.2 \mu \mathrm{m}$. The incident medium is air $\left(\eta_{m}=1\right)$ and the substrate is glass with $\eta_{s}=1.52$. The high- and the low-index refractive indices were $\eta_{h}=2.35$ and $\eta_{l}=1.35$, respectively. The merit function was defined at 39 points.

The initial number of layers is randomly chosen from 25 to 60 . The initial thickness of each layer was uniformly selected from the region from 0.01 to $0.1 \mu \mathrm{m}$. The maximum number of generations is set to 1500 .

The final designs are a 32-layer coating in the $s$-polarized case and a 59-layer coating in the p-polarized case for the short-wave-pass filter. Fig. 6 and Fig. 7 show the reflectance and refractive-index profiles of $s$-polarized case and p-polarized case, respectively. This problem seems to more difficult than long-wave pass filter for FCEA. The total thickness of the design in the p-polarized case is also 


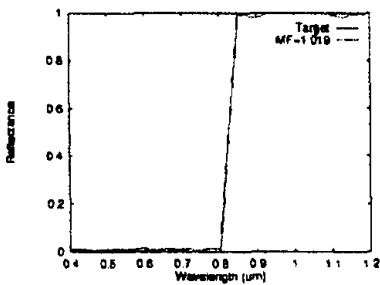

(a) Reflectance

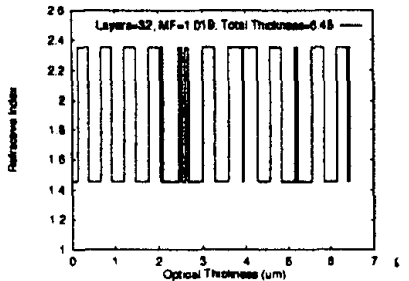

(b) Reflective index profile
Fig. 6. Reflectance and refractive-index profile of a 32-layer shortwave-pase filter for o-polarised light at a $45^{\circ}$ angle of incidence on interesting region $0.4 \leq \lambda \leq 1.2 \mu \mathrm{m}$ on a $\eta_{s}=1.52$ substrate based on the refractive index pair 1.45 and 2.35 .

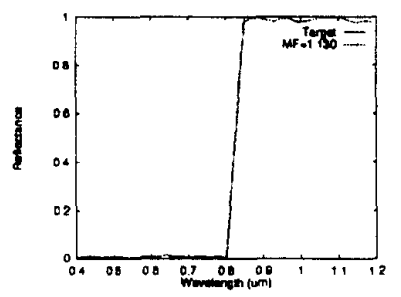

(a) Reflectance

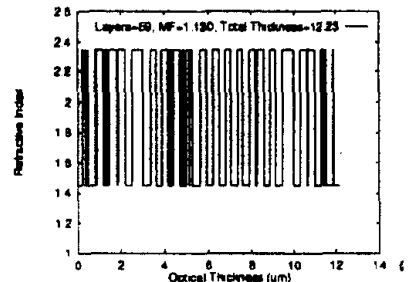

(b) Reflective index profile
Fig. 7. Reflectance and refractive-index profile of a 59-layer shortwave-pass filter for $p$-polarized light at a $45^{\circ}$ angle of incidence on interesting region $0.4 \leq \lambda \leq 1.2 \mu \mathrm{m}$ on a $\eta_{0}=1.52$ substrate based on the refractive index pair 1.45 and 2.35 .

considerably more than in the s-polarized case. The construction parameters of a s-polarized solution generated by FCEA are given in the second column of Table II for the short-wave-pass filter.

\section{Conclusions}

This study presents that FCEA is a stable synthesis approach for optical thin-film designs. Our experience suggests that a global optimization method should consist of both global and local search strategies. For our FCEA, the decreasing-based mutation with large initial step sizes is the global search strategy; the gelf-adaptive mutations with family competition procedure and replacement selection are local search strategies. Based on the family competition and adaptive rules, these mutation operators can closely cooperate with one another. Experiments of two difficult optical coating problems verify that the proposed approach is robust. We believe that the flexibility and robustness of FCEA make it an effective synthesis method of optical thin-film designs.

In future, we will investigate a more flexible approach to adapt the number of layers of a coating system. We will also consider a flexible mechanism to adapt the family competition lengths for improving the performance according to the performance improvement of mutations and the morphology of the landscape.

\section{REFERENCES}

[1] J. A. Aguilera, J. Aguilera, P. Baumeister, A. Bloom, D. Coursen, J. A. Dobrowolski, F. T. Goldstein, D. E. Gustafson, and R. A. Kernp. Antireflection coatings for germanium IR optics: a comparison of numerical design methods. Applied Optics. 27(14):2832-2840, 1988

[2] T. Blick. Evolutionary Algorithms in Theory and Practice. Oxford University Press, New York, USA, 1996.

[3] T. Bäck, U. Hammel, and H-P. Schwefel. Evolutionary computation: Comments on the history and current state. IEEE Trans. Evolutionary Computation, 1(1):3-17, 1997.

[4] T. Blick and H-P. Schwefel. An overview of evolution algorithms for parameter optimization. Evolutionary Computation, 1(1):123, 1993.

[5] K. Deb and R. B. Agrawal. Simulated binary crossover for continuous search epace. Complex Systems, 9:115-148, 1995.

[6] J. A. Dobrowolski. Optical properties of films and coatings. In M. Bass, editor, Handbook of Optics, chapter 42, pages 28242831. McGraw-Hill, New York, 1995.

[7] D. B. Fogel. Evolutionary Computation: Toward a New Philosophy of Machine Intelligent. NJ:IEEE Press, Piscataway, 1995.

[8] H. Greniner. Robust optical coating design with evolutionary strategies. Applied Optics, 36(28):5477-6482, 1996.

[9] L. Li and J. A. Dobrowolski. Computation speeds of different optical thin-film synthesis methods. Applied Optics, 31(19):3790$3799,1992$.

[10] H. A. Macleod. Thin film optical filters. McGraw-Hill, New York, 1986.

[11] S. Martin, J. Rivory, and M. Schoeanauer. Synthesis of optical multilayer systems using genetic algorithms. Applied Optics, 34(13):2247-2254, 1995.

[12] M. Schute and J. Sprave. Application of parallel mixed-integer evolutionary strategies with mutation rate pooling. In Proc. of the 5th Annu. Conf. on Evolutionary Programming, pages 345$354,1996$.

[13] H.-P. Schwefel. Numerical Optimixation of Computer Models. Chichester: Wiley, 1981.

[14] A. V. Tikhonravov. Some theoretical aspects of thin-film optics and their applications. Applied Optics, 32(28):5417-5426, 1993.

[15] A. V. Tikhonravov, M. K. Trubetsko, and G. W. Debell. Application of the needle optimization technique to design of optical coatings. Applied Optics, 35(28):5493-5508, 1996.

[16] J.-M. Yang, Y.-P. Chen, J.-T. Horng, and C.-Y. Kao. Applying family competition to evolution strategies for constrained optimization. In P. J. Angeline, R. G. Reynolds, J. R. McDonnell, and R. Eberhart, editors, Proc. of the 6th Annu. Conf. on Evolutionary Programming (Lecture Notes in Computer Science, vol. 1219), volume 1213, pages 201-211, 1997.

[17] J.-M. Yang and C.-Y. Kao. Flexible ligand docking using a robust evolutionary algorithm. Journal of Computational Chemistry, 21(11):988-998, 2000.

[18] J.-M. Yang and C.-Y. Kao. Integrating adaptive mutations and family competition into genetic algorithms as function optimizer. Soft Competing, 4(2):89-102, 2000.

[19] X. Yao and Y. Liu. Fast evolution strategies. In P, J. Angeline, R. G. Reynolds, J. R. McDonnell, and R. Eberhart, editors, Proc. of the 6th Annu. Conf. on Evolutionary Progromming (Lecture Notes in Computer Science, vol. 1219), pages 151-161, 1997. 